\title{
Aerodynamic Performance of Wind Microturbines and Their Dynamic Response
}

\author{
Gordon Breeze \\ BRE (Building Research Establishment), Watford WD25 9XX, UK
}

\begin{abstract}
Wind microturbines typically have rotor diameters of $2 \mathrm{~m}$ or less. This paper presents theoretical expressions that can be used to determine the aerodynamic performance of wind microturbines. A commercially-available three-bladed microturbine was tested at the outlet plane of a wind tunnel. The cross-section dimensions of the wind tunnel jet are $2.5 \mathrm{~m}$ (horizontal) $\times 1.5 \mathrm{~m}$ (vertical). The tested microturbine has a diameter of $1.2 \mathrm{~m}$, and it generates a maximum power output of about $300 \mathrm{~W}$. The paper provides the wind tunnel test methodology that was used to determine the mean and fluctuating forces generated by the aforementioned wind microturbine. Both the static and dynamic responses of the turbine were measured, and results from this testing are presented in this paper. These results enable the trends and predictions of the theoretical expressions to be compared with wind tunnel measurements. It is shown that, for this particular microturbine, the behaviours of these test measurements are consistent with the expected theoretical predictions.
\end{abstract}

Key words: Microturbine, wind, tunnel, aerodynamic, performance, forces, dynamics.

\section{Introduction}

Wind microturbines typically have rotor diameters of $2 \mathrm{~m}$ or less. For practical purposes, the wind generated forces acting on the mounting poles can be estimated using the method described in NHBC (National House Building Council) Foundation 2011 [1]; Technical details of the derivations are given in Ref. [2]. The method used to determine these wind-induced loads is based upon the codified approach described in BSI (British Standards Institution) [3], coupled with a dynamic magnification factor.

A commercially-available three-bladed microturbine (Fig. 1) was tested at the outlet plane of a BRE (Building Research Establishment) wind tunnel located in Garston, UK. The microturbine has a diameter of $1.2 \mathrm{~m}$, and it generates a maximum power output of about $300 \mathrm{~W}$. Within the rotor hub are springs which are attached by a linkage mechanism to the blade

\footnotetext{
Corresponding author: Gordon Breeze, principal consultant, research fields: wind engineering, industrial aerodynamics and vibration.
}

roots. These springs are designed to cause the blades to furl (i.e., change their angle of incidence) at a critical wind velocity, thus limiting the power output from the turbine. Measured power and the rotational characteristics of the microturbine are also shown in Fig. 1.

Work undertaken at BRE demonstrated that a microwind turbine mounted on a pole acts as a "1-DOF" (one degree of freedom) dynamic system. As shown below, the wind tunnel results can be combined with measurements of the dynamic loads acting on the mounting pole. This combination produces results and expressions which allows the mean and peak loads generated by wind microturbines to be predicted.

\section{Method Section}

The testing described in this paper was undertaken using BRE Wind Tunnel No. 3, which is located within a large building located at the BRE site. This large multi-purpose wind tunnel consists of a double centrifugal intake fan, which exhausts into a sealed settling chamber. Air passes from the settling chamber through a flow conditioning section, before exiting as a 


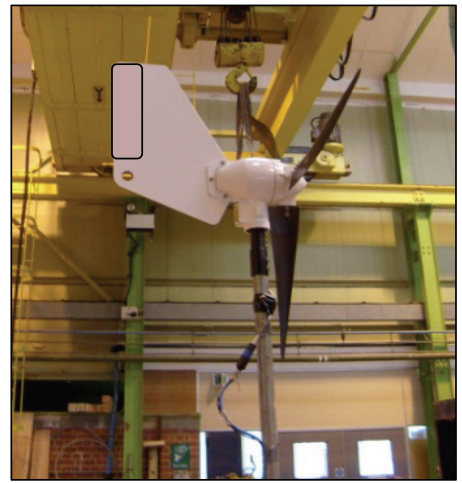

(a)

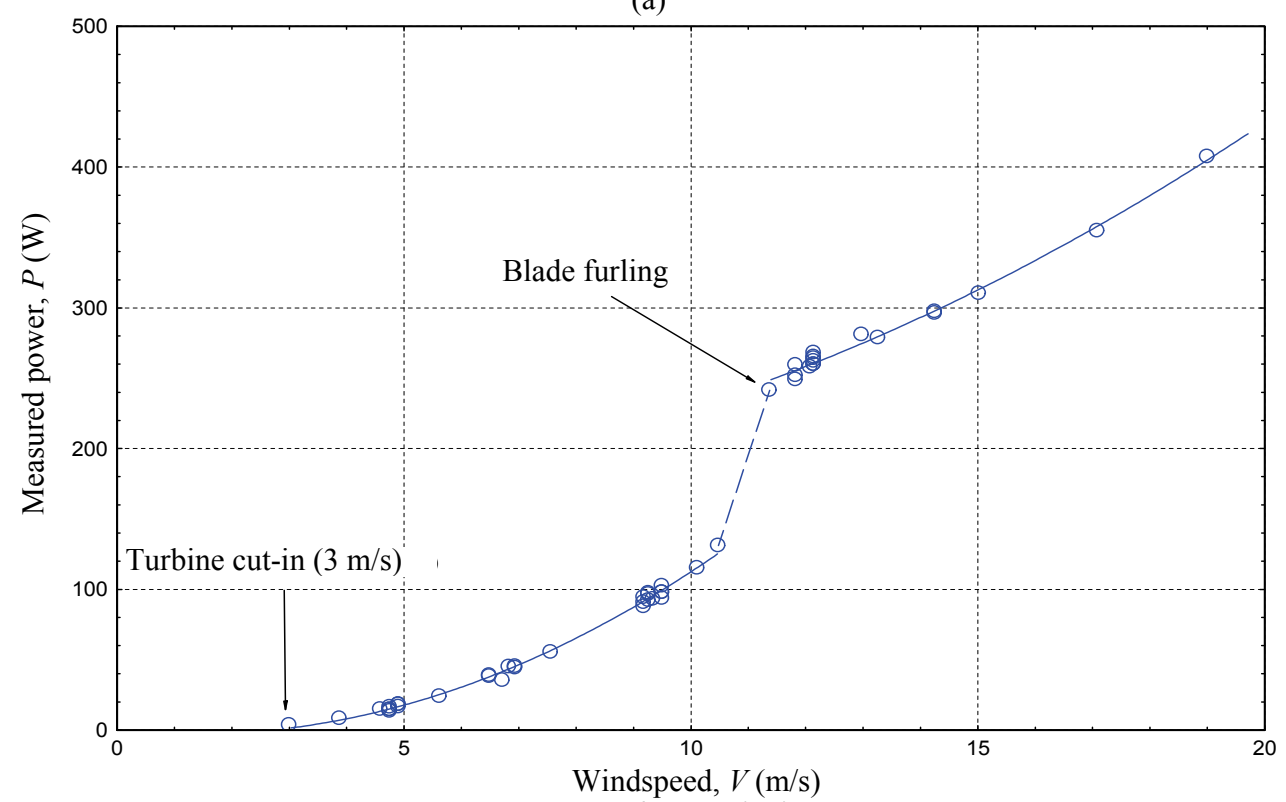

(b)

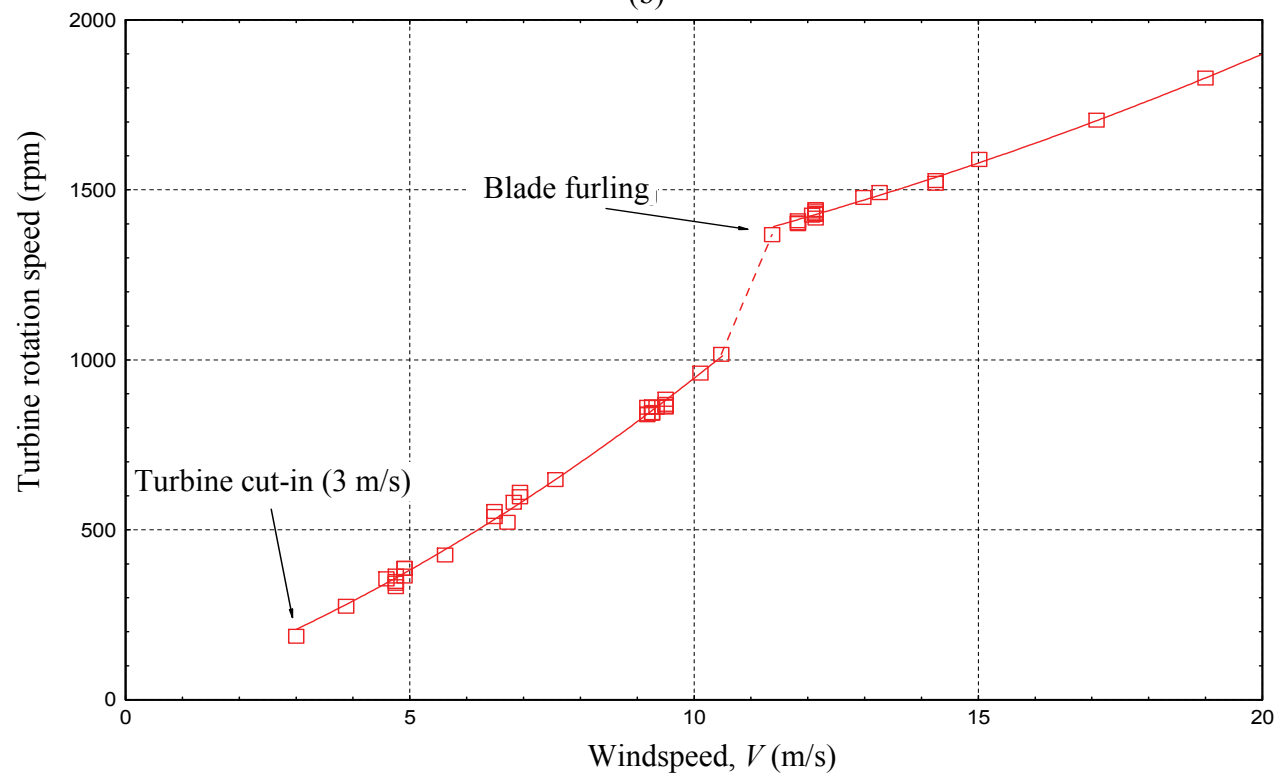

(c)

Fig. 1 The characteristics of the commercially-available wind microturbine: (a) photograph; (b) measured power; (c) rotational speed. 
high speed jet into the atmosphere. The wind tunnel can be configured in different ways. For these tests, the wind turbine was mounted $4.2 \mathrm{~m}$ from the outlet plane of the tunnel exit. At this outlet plane, the cross-section dimensions of the wind tunnel jet are $2.5 \mathrm{~m}$ (horizontal) $\times 1.5 \mathrm{~m}$ (vertical).

A photograph of the commercially-available wind microturbine is shown in Fig. 1. The electrical output from windings around the turbine stator consists of three-phase voltages. The windings contain 12 poles, or six pole-pairs, the significance of which is described below. The three-phase output voltages from the turbine were rectified, and the rectified voltages attached to a $19.2-\Omega$ resistive load. The voltage across the resistive load and the current passing through the load were measured, using a calibrated multi-meter and ammeter. The power generated by the turbine was calculated by taking the product of the measured DC (direct current) amps and volts.

The wind microturbine was mounted on top of a scaffold pole located at the outlet of the wind tunnel. The external diameter of this pole was $0.0483 \mathrm{~m}$, and the structural rigidity $(E I)$ of the pole was $2.898 \times 10^{4} \mathrm{Nm}^{2}$. The length of the scaffold pole $(2.38 \mathrm{~m})$ was cut to allow the turbine to be positioned in the centre of the outlet jet. Below the wind tunnel jet, the base of the pole was attached (using scaffold clamps) to a horizontal beam that was fixed at its ends to two posts located on either side of the wind tunnel.

The aim of the experimental arrangement was that wind-induced vibrations from the wind turbine would be detected by the along-wind movement of the centre of the horizontal beam. Since the scaffold pole is attached rigidly to the horizontal beam, the time-varying along-wind movement (or displacement) of the beam is the same as the movement of the mounting bracket. Before the testing was undertaken, the stiffness of the horizontal beam was determined. By measuring the beam displacement and knowing the beam stiffness, at the pole mounting bracket location, the time-varying wind-induced forces could then be calculated.

The displacement of the centre of the horizontal beam was measured using a laser system. The laser system was positioned on the ground downstream of the horizontal beam, at a location where the system was outside of the air jet emanating from the wind tunnel. The laser beam was focussed at the centre of the horizontal beam - as noted above, the pole mounting bracket was attached to the opposite side of this beam. For this experiment, the laser system was set to measure displacement. Lasers measure displacement only in the direction of the laser light, and the readings from the laser were corrected to take into account the downward inclination angle of the laser head.

The rotational speed of the microturbine was obtained by measuring the frequency of the voltage signal between two of the turbine windings. Since there are six pole-pairs and $60 \mathrm{~s}$ in a minute, the rpm (revolutions per minute) of the turbine was 10 times the frequency $(60 / 6)$ of the measured voltage signal.

To obtain suitable signals for subsequent analysis, the voltage output from the laser (which is proportional to the displacement) and the voltage between the turbine windings were low-pass filtered at $200 \mathrm{~Hz}$, and amplified using Kemo Dual Variable Filter Type $\mathrm{VBF} / 8$ (Attenuation 48bB/Octave) analogue filter/amplifiers. Both of these voltages were sampled simultaneously at $400 \mathrm{~Hz}$ using a calibrated Cambridge Electronic Design CED1401A/D device. This device was controlled by means of a propriety data acquisition and analysis program, and the data acquired written directly to an Excel spreadsheet. Each record contained 5,000 samples, and the length of time of each record was, therefore, $12.5 \mathrm{~s}(5,000 \div 400)$.

The wind tunnel was set at a range of speeds with the power performance of the turbine and the beam displacement measured at each wind tunnel setting.

${ }^{1}$ For such investigations, it is essential to minimise "aliasing" of the frequency signal. The incorporation of the low-pass filters ensures that the frequency domain analysis does not contain unwanted "aliased" contributions coming from higher frequencies. 
It is noted that the wind tunnel setting speed is not the same as the windspeed at the wind turbine measurement position. Before the testing was performed, a velocity traverse across the turbine plane was undertaken. This showed that, to obtain the windspeed at the turbine, the measured tunnel speed (measured at the wind tunnel outlet) needed to be factored by 0.95 . In this paper, all of the wind tunnel speeds have been corrected in this way.

\section{Static Results}

The efficiency of a turbine system is defined in Ref. [4] by the following equation:

$$
\eta=\frac{P}{\frac{1}{2} \rho A V^{3}}
$$

where, $P$ is the power generated by the turbine (W), $A$ is the swept area of the turbine rotor $\left(\mathrm{m}^{2}\right), \eta$ is the overall efficiency of the turbine and its associated electrical equipment (i.e., the overall efficiency of the system), $V$ is the windspeed $(\mathrm{m} / \mathrm{s})$ and $\rho$ is the air density $\left(\mathrm{kg} / \mathrm{m}^{3}\right)$.

The electrical power output was measured in the wind tunnel at a range of speed settings. This information was used to calculate the overall efficiency of the wind microturbine system $(\eta)$; The results are shown as the black trace in Fig. 2. It can be seen that the measured maximum efficiency occurs at about $11 \mathrm{~m} / \mathrm{s}$ - the speed at which the blade furling starts to occur. It can be seen that the efficiency is reasonably independent of windspeed and varies between $16 \%$ and $23 \%$.

The average efficiency of the data presented in Fig. 2 is 0.187 ; The corresponding coefficient of determination $R^{2}$ is 0.23 .

The overall efficiency of the system $\eta$ can be expressed as the product of the electrical and aerodynamic efficiencies ( $\eta_{e}$ and $\eta_{a}$, respectively), viz: $\eta=\eta_{e} \cdot \eta_{a}$

The variable $\eta_{a}$ accounts for the aerodynamic efficiency of the rotor and is, therefore, the difference between unity and the ratio between the non-recoverable aerodynamic pressure losses (caused by drag, flow swirl and turbulence), and the total dynamic pressure $\left(1 / 2 \rho V^{2}\right)$ of the approaching flow.

The variable $\eta_{e}$ accounts for electrical losses. Therefore, $\eta_{e}$ is the difference between unity and the ratio between the sum of: (1) the electrical power losses of the AC (alternating current) 3-phase generator and cabling losses; (2) the 3-phase to DC rectification losses, and the overall power $P$ generated by the turbine.

The non-dimensional thrust coefficient $C_{T}$ has also been plotted as the red trace of Fig. 2. This coefficient is defined by the following equation:

$$
C_{T}=\frac{P^{2}}{\left(\frac{1}{2} \rho V^{2}\right)^{2} \times A \times V^{2} \times B(c R)}
$$

where, $B$ is the number of turbine blades $(B=3), R$ is the turbine blade radius $(R=0.6 \mathrm{~m})$ and $c$ is the average turbine blade chord $(c=0.082 \mathrm{~m})$.

It can be shown that the thrust coefficient is related to the force coefficient $C_{F(t u r b)}$ by the Eq. (3):

$$
C_{T}=\eta_{e} \times C_{F(t u r b)}
$$

where, $\eta_{e}$ is the aforementioned electrical efficiency of the turbine generation system. Fig. 2 shows that $C_{T}$ varies systematically over the tested windspeed range. However, this variation is not large and the average value over the range of windspeeds tested $(3 \sim 19 \mathrm{~m} / \mathrm{s})$ is 0.28 . Using this average value, the corresponding coefficient of determination $R^{2}$ is 0.18 .

If the efficiency of the electrical system is reasonably constant over the range of power being generated (which is not an unreasonable assumption), Eq. (3) shows that $C_{F(t u r b)}$ must also be relatively insensitive to changes of windspeed.

\section{Dynamic Results}

For a 1-DOF wind microturbine and pole mounting system vibrating at resonance, the amplitude of the pole mounting forces $F_{0}$ is given by Eq. (4): 


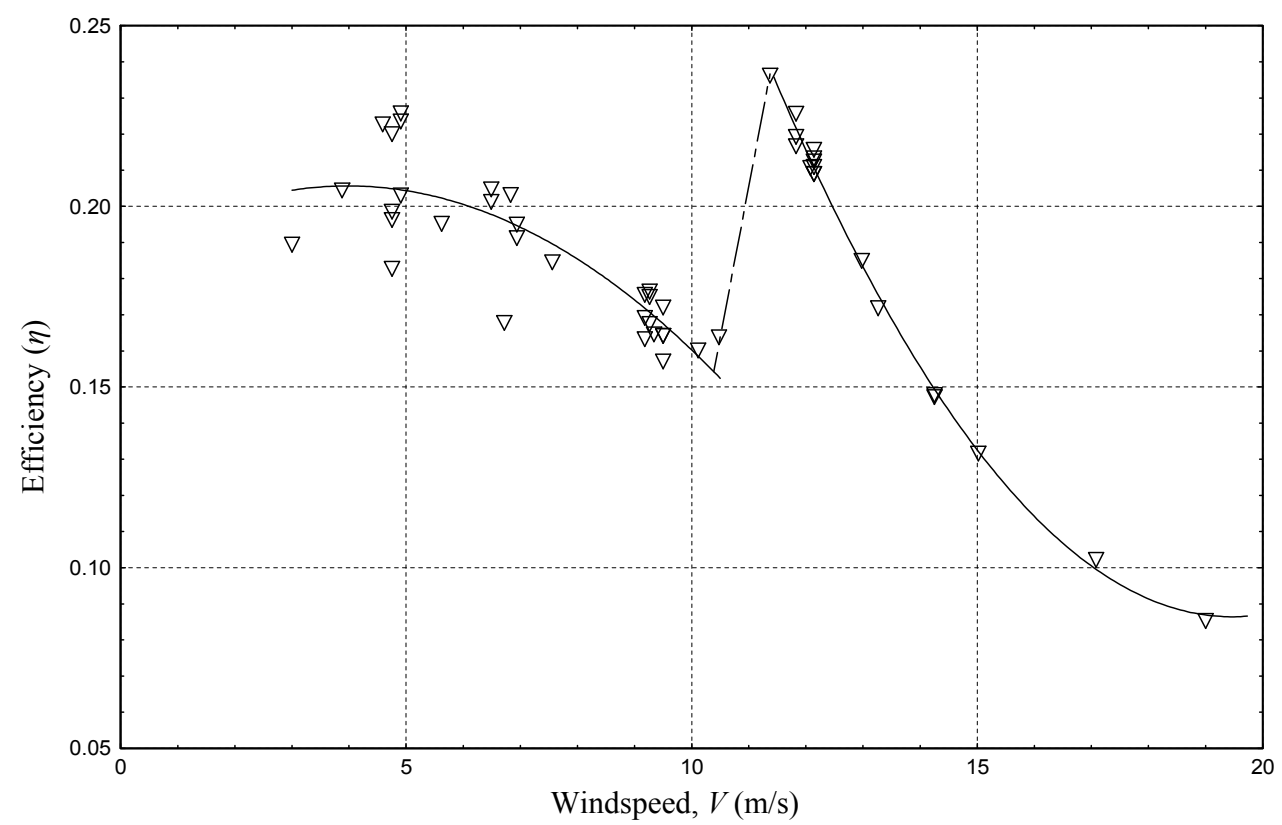

(a)

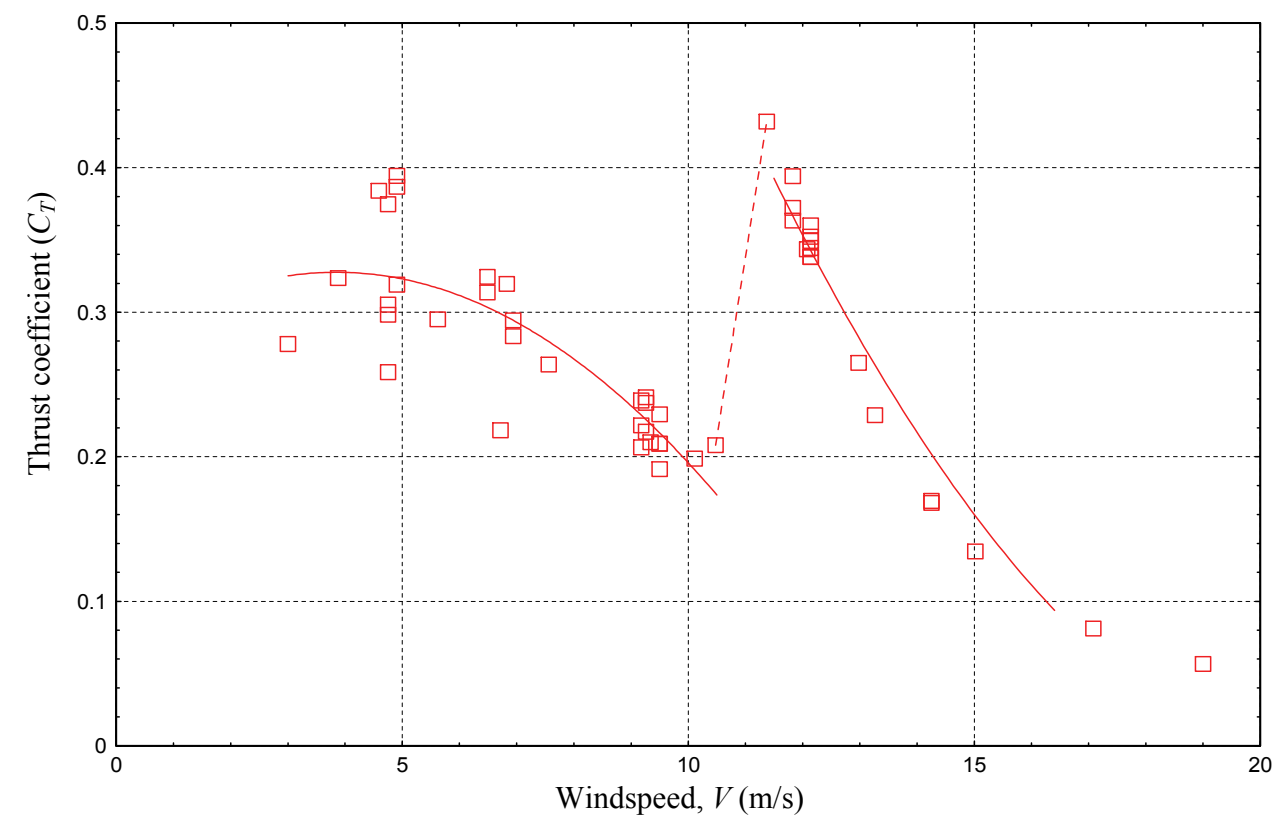

(b)

Fig. 2 Variations with windspeed: (a) efficiency; (b) thrust coefficient.

$$
F_{0}=\frac{\left(\frac{1}{2} \rho V^{2}\right) \times\left[B(c R) C_{F(\text { turb })}+(L d) C_{D}\right]}{2\left\{\varsigma_{s}+\varsigma_{b}+\varsigma_{a}\right\}}=\frac{\left(\frac{1}{2} \rho V^{2}\right) \times\left[B(c R) C_{F(\text { turb })}+(L d) C_{D}\right]}{2\left\{\varsigma_{s}+\varsigma_{b}+\frac{\left(2 \pi f_{0}\right)}{2} \times V \times \rho \times\left(\frac{L^{3}}{3 E I}\right) \times\left[B(c R) C_{F(\text { turb })}+(L d) C_{D}\right]\right\}}
$$

where, $L$ is the exposed length $(\mathrm{m}), d$ is the diameter (m), $C_{D}$ is the drag coefficient of the mounting pole, $\varsigma_{s}$, $\varsigma_{b}$ and $\varsigma_{a}$ are respectively the critical damping coefficients of the structure, the turning blades and the static blades, $f_{0}$ is the fundamental natural frequency of the system $(\mathrm{Hz})$, and $E I$ is the structural rigidity of the mounting pole $\left(\mathrm{Nm}^{2}\right)$. This expression can be derived simply from the 1-DOF equations of motion (at resonance) given in many dynamics textbooks, e.g., Ref. [5]. 
With the wind tunnel not running, the structural damping ratio $\left(\varsigma_{s}\right)$ was determined by measuring the logarithmic decay of the pole oscillations.

From Eq. (4), the following equation can be seen:

$$
\begin{gathered}
\varsigma_{a}=\frac{\left(2 \pi f_{0}\right)}{2} \times V \times \rho \times\left(\frac{L^{3}}{3 E I}\right) \times \\
{\left[B(c R) \times\left(\frac{C_{T}}{\eta_{e}}\right)+(L d) C_{D}\right]}
\end{gathered}
$$

It should be noted that $\varsigma_{a}$ is not proportional to $V$, since $C_{T}$ varies with $V$, as shown in Fig. 2. For the test arrangement shown in Fig. $1, f_{0}=2.6 \mathrm{~Hz}, E I=2.898 \times$ $10^{4} \mathrm{Nm}^{2}, L=2.38 \mathrm{~m}, d=0.0482 \mathrm{~m}$, and $C_{D}=1.2$.

The time-varying forces generated by the microturbine were measured using a dynamic force balance, and the spectra of these forces were calculated using an FFT (fast Fourier transform) algorithm. For a 1-DOF dynamic system, the force-displacement gain factor $\left|H(f)_{f-d}\right|$ is given in Ref. [6]:

$$
\left|H(f)_{f-d}\right|=\frac{(1 / k)}{\sqrt{\left[1-\left(\frac{f}{f_{0}}\right)^{2}\right]^{2}+\left[2 \varsigma\left(\frac{f}{f_{0}}\right)\right]^{2}}}
$$

where, $k$ is the stiffness of the system, and $\varsigma$ is the system damping ratio.

Using this expression, the turbine system damping $\varsigma_{b}$ was the value which corresponded with the best fit of the measured spectra at frequency $f_{0}$.

Values of the aerodynamic damping $\left(\varsigma_{a}\right)$ were calculated using Eq. (5) for a range of electrical efficiency $\left(\eta_{e}\right)$ values.

For a range of wind tunnel speeds, the amplitudes of the fluctuating forces acting on the turbine pole $\left(F_{0}\right)$ were measured using the aforementioned force balance, and the results are shown in Fig. 3. The black line shown in this figure was calculated using Eq. (4), with an electrical efficiency $\left(\eta_{e}\right)$ of 0.324 (32.4\%); This electrical efficiency gave the best fit with the experimentally measured data. The coefficient of determination $R^{2}$ of the line shown in Fig. 3 is 0.69. Although Eq. (4) shows that there is actually a complex relationship between $F_{0}$ and windspeed $V$, Fig. 3 shows that, for practical purposes, the amplitudes of the fluctuating forces are proportional to the approaching windspeed $V$.

With the wind tunnel running, it was observed that the amplitude of the force acting on the turbine pole varied in time. The variation of the force amplitude was quantified by measuring its standard deviation $\sigma_{f}$. The variation of $\sigma_{f}$ with windspeed is shown in Fig. 4; It can

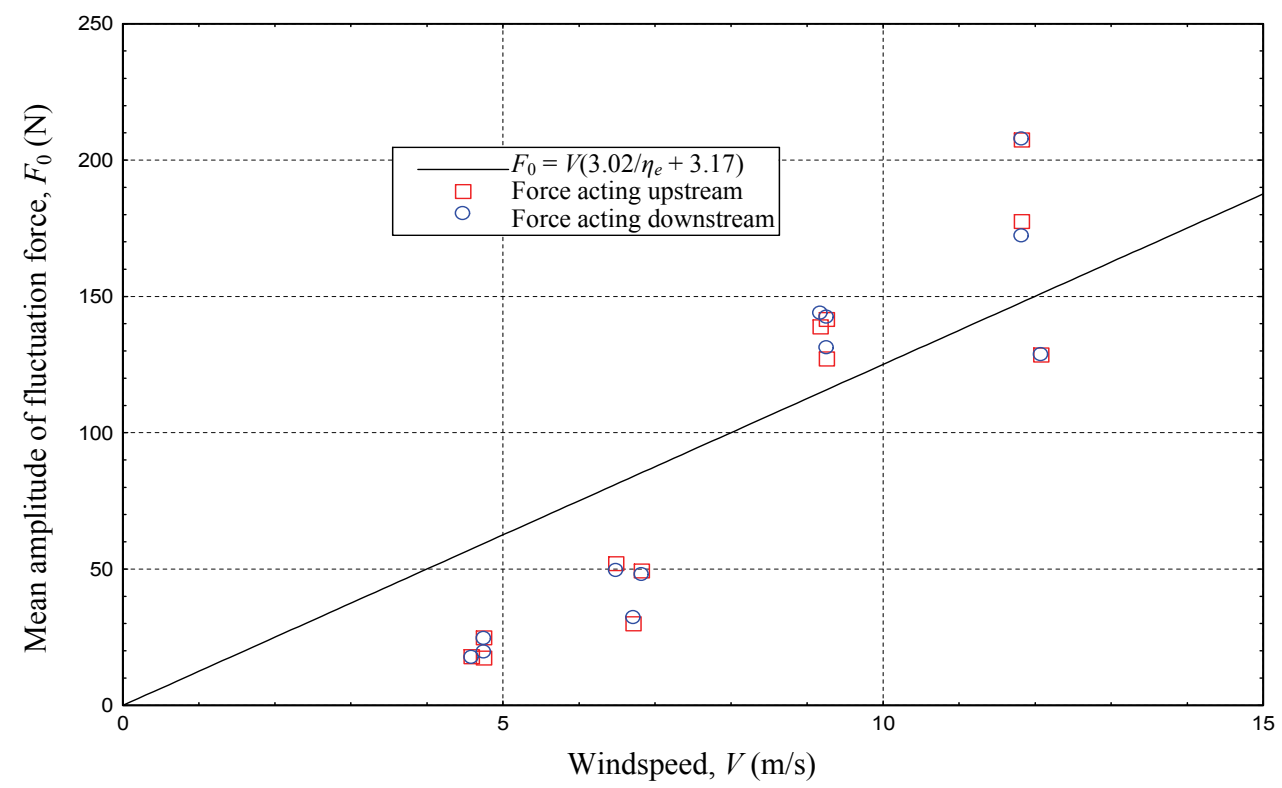

Fig. 3 Variation of fluctuating force amplitude with windspeed. 


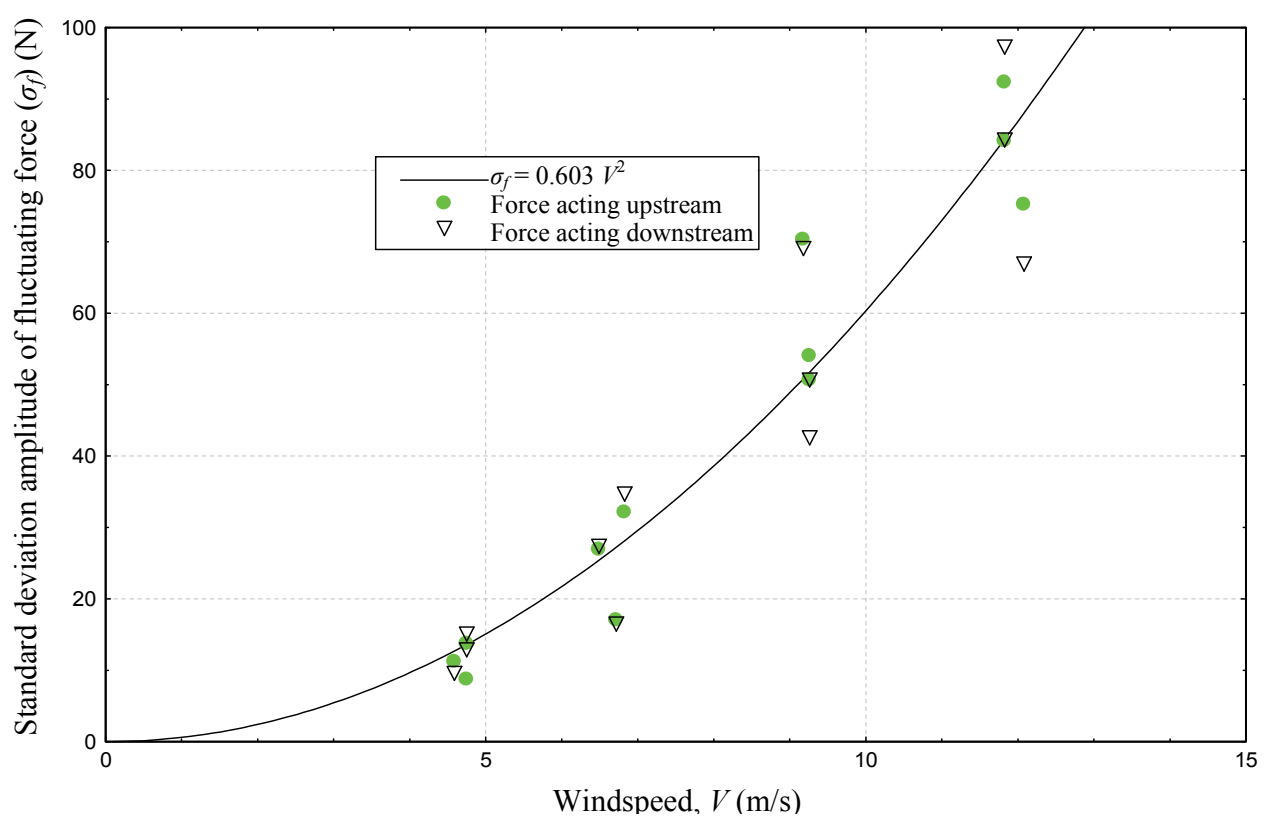

Fig. 4 Variation of standard deviation of fluctuating force amplitude with windspeed.

be seen that this parameter increases with the square of the speed. The coefficient of determination $R^{2}$ of the shown curve is 0.90 .

Using a peak factor approach, the peak wind load produced by the microturbine can be estimated using Eq. (4), and the empirical expression shown in Fig. 4.

\section{Conclusions}

Equations are presented in this paper that enables the mean and fluctuating forces generated by a wind microturbine to be predicted. These equations have been derived from well-established aerodynamic and structural engineering physical principles, and they are presented in a way that shows explicitly the pertinent aerodynamic and structural parameters.

Wind tunnel testing of a commercial wind microturbine was undertaken, the results of which are presented in this paper. It is shown that, for this particular microturbine, the behaviours of these test measurements are consistent with the expected theoretical predictions.

It is not possible to draw general conclusions from the obtained results from testing a single microturbine design. This is especially the case for the tested microturbine because of blade furling mechanism which might be unique to this particular design. Nevertheless, the fact that the test results are consistent with the theoretical equations means that the expected behaviour of other microturbines can be predicted with a reasonable level of confidence.

\section{References}

[1] NHBC (National House Building Council) Foundation. 2011. Guide to Installation of Renewable Energy Systems on Roofs of Residential Buildings, NF 30. Watford: BRE (Building Research Establishment) Press.

[2] Breeze, G. 2012. "Static and Dynamic Wind Loads on Building-Mounted Microwind Turbines." In BRE Information Paper IP 14/12. Watford: BRE.

[3] BSI (British Standards Institution). 2006. Wind Turbines-Design Requirements for Small Wind Turbines. BS EN 61400-2:2006. London: BSI.

[4] Duncan, W. J., Thom, A. S., and Young, A. D. 1970. Mechanics of Fluids. 2nd ed. Delft: Arnold.

[5] Clough, R. W., and Penzien, J. 2003. Dynamics of Structures. 3rd ed. New York: Computers and Structures Inc.

[6] Bendat, J. S., and Piersol, A. G. 1971. Random Data: Analysis and Measurement Procedures. New York: Wiley Inerscience. 